\title{
Defending The Semantic View: What It Takes
}

\author{
Soazig Le Bihan*
}

October 31, 2008

\begin{abstract}
In this paper, a modest version of the Semantic View is motivated as both tenable and potentially fruitful for philosophy of science. An analysis is proposed in which the Semantic View is given as characterized by three main claims. For each of these claims, a distinction is made between stronger and more modest interpretations. It is argued that the criticisms recently leveled against the Semantic View hold only under the stronger interpretations of these claims. However, if one only commits to the modest interpretation for all the claims, then the view obtained, which is called the Modest Semantic View, is tenable and fruitful.
\end{abstract}

\section{Introduction}

Since the seventies, the Semantic View has gradually become the new orthodoxy in philosophy of science. ${ }^{1}$ As such, it is both widespread and heavily

*University of Montana, www.soaziglebihan.org. I wish to thank Guido Bacciagaluppi, Michael Dickson, and Armond Duwell for substantive and insightful comments on earlier drafts of this paper.

${ }^{1}$ Major developments of the Semantic View are in Suppes (1960, 1962, 1967), Suppe (1974), Giere (1979, 1988), van Fraassen (1980, 1989, 1991), Suppe (1974, 1977, 1989). Van Fraassen (1980, Chapter 3, p. 64, note 22) gives references to previous works outside of the English-speaking world, mainly in Poland and Italy (Wójcicki, dalla Chiara). Suppe provides an erudite account of the historical development of the Semantic View as originating in the views of Beth $(1948 \mathrm{a}, 1948 \mathrm{~b}, 1949,1960)$ in the preface of his $(1989$, p. 5-20). In their subsequent works, all these authors typically remained in favor of the Semantic View broadly speaking, even if each developed a particular version of it and never formed 
criticized. $^{2}$ In this paper, it is argued that the criticisms which have been leveled against the Semantic View in the literature are both fair and unfair. On the one hand, they are unfair because they rely on strong interpretations of the main claims of the Semantic View, strong interpretations to which it will be argued the Semantic View does not have to commit. On the other hand, the criticisms are fair, because the various advocates of the Semantic View generally fail to explicitly recognize the distinction between the stronger and the more modest interpretations of their claims. In this paper, these distinctions are clarified and a modest version of the Semantic View is motivated as both tenable and fruitful.

The Semantic View can be seen as being constituted by the three following main claims ${ }^{3}$ :

1. (Models) - Scientific theories and scientific practice can be studied through the scientific models that scientists typically use to represent the world;

2. $($ Scientific $=$ Logical $)-$ Scientific models can be construed as logical models;

3. $(\rightarrow$ Adequacy $)$ - Studying scientific theories by studying their models and construing scientific models as logical models provides the means to give an adequate account of what scientists typically use to represent the world in actual practice.

a "school" in any rigorous sense. The school founded by Sneed (1971), and developed by Stegmüller $(1976,1979)$ and Moulines (1975) is typically considered as having become a trend of its own, called the "non-statement view" or the "structuralist school". One classic work for the structuralist school is Balzer et al. (1987). Other important contributions to the "classic" Semantic View in the biological domain are Lloyd (1994)[1988], Thompson (1989) and the publications by Beatty including his $(1979,1980)$. One beautiful and often forgotten book in the field is Hughes (1989). One important recent work in which the Semantic View is used is da Costa and French (2003).

${ }^{2}$ See Redhead (1980), Cartwright (1983, 1989, 1999), Ereshefsky (1991), Morrison (1999), Suarez (2003), Thomson-Jones (2006), Frigg (2006), Morrison (2007), and Bueno and Krause (2008).

${ }^{3}$ What follows is a conceptual reconstruction of the Semantic View. No pretension is made to give a historically adequate account of how the Semantic View developed. Note also that the differences between the set-theoretical approach of Suppes, the state-space approach of van Fraassen and the relational system approach of Suppe are irrelevant here. We agree with Suppe (1989, p. 4) and da Costa and French (2003, p. 23) that van Fraassen's and Suppe's approaches can be conceived within Suppes' set-theoretical framework. For this reason, Suppes' approach is discussed in this paper. 
Each of these three claims have been criticized in the recent literature. In the following sections, each of these three claims together with their criticisms will be analyzed in turn. In each case, it will be shown that 1 . each of these three claims can be interpreted either in stronger or in more modest manners; 2. the criticisms leveled against each these claims in the literature hold only under the strong interpretations of the claims. This implies that:

- if one commits to any of the strong interpretations of any of the three claims, then the criticisms hold and the Semantic View fails;

- if one does not commit to any of the strong interpretations of any of the three claims, and restricts her commitment to the most modest interpretation instead, then the criticisms fail and the view obtained, which is called here the Modest Semantic View, is tenable.

In the last section of the paper, it will also be explained in what sense and to what extent the Modest Semantic View is not only tenable but also is a fruitful tool for philosophy of science.

\section{2 (Models): Theories, Models and Scientific Practice}

It is well known that advocates of the Semantic View have urged philosophers of science to 1. stop studying scientific theories from the point of view of their laws or their axiomatic structure, and 2. to focus on scientific models instead. They typically justify this recommendation in the following way.

First, advocates of the Semantic View urge philosophers of science to stop studying scientific theories from the point of view of laws and axioms because, according to them, such a method results in philosophers of science neglecting a large portion of well-recognized scientific practice. ${ }^{4}$ They recognize that some scientific theories can be construed as sets of laws and that, for

\footnotetext{
${ }^{4}$ To conceive of scientific theories as set of laws or of axioms was part of what has been labeled the "received view" by Putnam in his (1962) because it has been the major trend in philosophy of science for more than half a century. It is not clear that the label refers to a single unified view rather than to a variety of positions taken at different times by various authors (For a presentation of the historical development of the received view, see for example Friedman (1999) or Parrini et al. (2003)). That said, the view is generally taken to go along the following lines (see Carnap (1966), Hempel (1963)). According to the received view, or the Syntactic View, a scientific theory is defined as an axiomatic
} 
these theories that are axiomatizable, working on possible axiomatizations can give important insights on the structure of these theories (see for example Suppes (2002 p. 28-30), van Fraassen (1980 p. 64), Lloyd (1994, p. 8)[1988]). That said, the domain of philosophy of science cannot be restricted to axiomatizable theories, simply because most of the scientific theories that are used in scientific practice are not axiomatizable, as Suppes strongly states (2002, p. 27):

A major point I want to make is that a simple standard formalization of most theories in the empirical sciences is not possible.

Suppe (1977 pp. 62-66) provides an analysis of what counts as a fruitful axiomatization, and a long list of well-accepted and widely used scientific theories which do not admit of any fruitful axiomatization. Neuroscientific or paleontological theories, for example, cannot be formulated through a nice set of axioms. Further, it is not even clear that all scientific theories include "laws" in any robust sense. One important reason why Lloyd (1994)[1988] favors the Semantic View for studying evolutionary theory is precisely that, in focusing on the models used, the Semantic View allows one to avoid the conclusion that biological theories do not count as scientific theories even if there are no biological laws. Neuroscientific, paleontological and biological theories are well-accepted and widely used in actual scientific practice. As such, they are part of the proper domain of philosophy of science. That they are theories that are not axiomatizable or which do not feature universal laws should not lead one to conclude that these theories are not scientific. Instead, as Lloyd claims (1994, p. 7)[1988]:

[... the inability of the received view to take evolutionary theory into account is a flaw of [the axiomatic] approach.

In short then, to conceive of scientific theories as set of laws or as sets of axioms is too restrictive.

system of formal sentences, which are formulated in a theoretical language, and partially interpreted in terms of an observational language, by means of rules of correspondence. Such a definition functions as a normative criterion of demarcation between science and pseudo-science. The Syntactic View has been criticized along various lines. In particular, advocates of the Semantic View heavily criticized the Syntactic View for its linguistic orientation. That said, there will be no need for a precise account of these criticisms for this paper. Suppe provides an extensive treatment of this issue in his (1989, part I). 
As a remedy to the problem above, a second move is proposed, namely to focus on scientific models. An important motivation for this is the factual observation that scientists use scientific models to represent the world. ${ }^{5}$ In Newtonian physics for example, one uses the two gravitational body system with point masses, the block moving down a slope, and the harmonic oscillator to represent the Earth orbiting the Sun, two kids sledding in the winter or our grandmothers' clocks, respectively. That said, scientific models are not only used for mature physical theories. Models are used throughout the scientific realm, including for example the computational model of the brain in neuroscience, the Lotka-Volterra models of predator-prey interactions, or the well-known helix model of DNA. To quote Lloyd concerning evolutionary biology (Lloyd 1994, p. 9)[1988]:

The main reason that models should be considered in any description of the structure of evolutionary theory is that models themselves are the primary theoretical tools used by evolutionary biologists. Biologists often present their theories in terms of models, and they often draw conclusions using these models. ${ }^{6}$

In short then, it seems that by studying scientific models, one in fact studies what scientists typically use to represent the world in actual practice. This factual observation about scientific practice is one of the main motivations for (Models), the first of the three claims which together constitute the Semantic View:

(Models) - Scientific theories and scientific practice can be studied through the scientific models that scientists typically use to represent the world;

\footnotetext{
${ }^{5}$ It is well known that there are various kinds of 'scientific models'. No stance is taken in this paper as to any particular mode of representation (structural, analogous, iconic etc.) by which scientific models represent the world. For a synthesis of the notions of model in science, see Frigg and Hartman (2006).

${ }^{6}$ Suppe (1977a, p. 222) and van Fraassen (1989, p. 211 and p. 224-225) also argue that the Semantic View is giving an account closer to actual practice. A classical argument formulated by advocates of the Semantic View is that scientific theories are typically formulated in terms of models in textbooks (sometimes despite the appearances): see Beatty (1979, pp. 95-98) for evolutionary theory, van Fraassen (1980, p. 65) for quantum mechanics, and Giere (1988, chap. 3) for classical mechanics.
} 
In its most modest interpretation, (Models) includes the following partial, descriptive statement about scientific practice: scientists typically use scientific models to represent the world. Now, there are at least three other ways in which such a statement can be interpreted. ${ }^{7}$ First, (Models) can be interpreted as including a complete account of scientific practice. In this case, it is interpreted as including the claim that all scientific practice is the use of scientific models to represent the world. A second, stronger interpretation is to interpret (Models) as including a complete view of scientific theories, i.e. the view that scientific theories are but sets of models. On top of this, (Models) can be interpreted as including a normative claim about scientific theories and scientific practice. In this case, (Models) is interpreted as including the claim that only these theories that are used to represent the world through scientific models are scientific theories. It should be clear that none of these interpretations necessarily follows from (Models) when seen as only including a descriptive and partial characterization of scientific practice.

The normative interpretation is the most easy one to reject in the context of the Semantic View. Advocates of the Semantic View have consistently rejected the normative interpretation of (Models) (see for example Suppe $(1979$, p. 322)). The Semantic View was never intended to provide a normative criterion for demarcation. Instead, it is intended to provide a descriptive account of what scientists use to represent the world in actual practice. Advocates of the Semantic View recommend that the issue of demarcation be addressed outside of formal methods. Their suspicion is that there can be no formal characterization of what makes a scientific theory scientific. The demarcation problem is thus not part of the issues that the Semantic View is intended to address. Instead, advocates of the Semantic View take as their object of study whatever models scientists agree on to use to represent the various domains of science.

Advocates of the Semantic View are much less explicit about whether or not they take (Models) as including a complete view of scientific theories. That said, any unbiased reader will find plenty of evidence showing that the modest interpretation is the one which is favored. Attention should be paid to the vocabulary used when it comes to relate theories and models. Van Fraassen almost systematically uses the vocabu-

\footnotetext{
${ }^{7}$ The distinctions made in the following are related, but not identical to the distinctions between intrinsic and extrinsic characterization of theories by Suppes (1967, p. 60-62, and 2002), and between representational and constitutional role of models by da Costa and French (2003 p. 34).
} 
lary of exposition/presentation by contrast to a vocabulary of identification/definition/reduction when it comes to link 'theories' with 'sets of models' (1980 p. 64):

To present a theory is to specify a family of structures, its models $[\ldots]$.

Notice that van Fraassen does not claim that theories are either defined by, identifiable with, even less reducible to, sets of models. He remains faithful to this usage in his later works. All that he claims is that theories can be presented through their sets of models. ${ }^{8}$ Lloyd is also consistent in her use of a vocabulary of presentation/exposition instead of definition/identification/reduction. As to Suppe, he claims that, according to the Semantic View, "the heart of a theory is an extralinguistic theory structure" which can be "characterized" or "specified" by a class of intended models (Suppe, 1989 p. 4). Suppes is even more explicit when it comes to rejecting the idea that the Semantic View gives a definition of scientific theories (2002, p. 8):

It does not seem important to me to give precise definitions of the form: $X$ is a scientific theory if, and only if, so-and-so. ${ }^{9}$

Granted, some may be less cautious, as Giere, in the following (1988, p. 48):

This makes possible to identify a theory [...] with the set of models that would be picked out by all the different possible formulations.

That said, it would be mistaken to conclude that for Giere, a theory reduces to a set of models, given that he also claims that scientific theories include as an essential part a "theoretical hypothesis" by which the models of the theory are related to the world $(1988$, p. 65$)$. The upshot is that there is not

\footnotetext{
${ }^{8}$ One possible exception is in his $(1989$, p. 222$)$ where he maintains that if we have to identify a theory with something, then it is better to identify it with the class of its models than with a set of sentences. That said, the qualifications that van Fraassen puts on his statement are clear enough to show that he is not committing to a definition of theories as sets of models.

${ }^{9} \mathrm{~A}$ few pages earlier, Suppes warns his reader that the question "what is a scientific theory?" is not the type of question to which we should expect to give a precise and definite answer.
} 
much doubt that the Semantic View was never committed to the claim that a theory is nothing but a set of models. That said, the point is typically not made perfectly explicit.

One last point has to be made regarding the possible interpretation of (Models) as including the claim that scientific practice consists only in the use of models for representation. It has to be granted that advocates of the Semantic View sometimes seem to fall on the side of this strong interpretation. The following is a quote from the young van Fraassen $(1972$, p. 310).

[...] the essential job of a scientific theory is to provide us with a family of models, to be used for the representation of empirical phenomena.

In addition, there have been numerous claims that the Semantic View allows philosophers of science to get closer to scientific practice. This could be seen as implying that scientific practice is only the use of models to represent the world. Such claims, however, have to be put in context. The context of the development of the Semantic View was the failure of the Syntactic View, which was criticized for having lost sight of its proper domain. As Suppe puts it, the discussions within the Syntactic View "did not have much to do with real science (1989, p. 14)". Considering directly the class of models used to represent a given domain allowed one to avoid the linguistic issues that had plagued philosophy of science, such as the distinction between theoretical and observational terms for example. Now, to point toward scientific models as essential tools in scientific practice is not necessarily claiming that all scientific practice is but the use of models for representation. It is unlikely that advocates of the Semantic View ever did interpret (Models) as including the latter claim. That said, one has to grant that, as in the two previous cases, this is typically not made perfectly explicit.

It is crucial, however, to make the distinctions explicit and to commit only to the modest interpretation of (Models), simply because the strong interpretations of (Models) are easily shown to be false. Against the claim that scientific practice is limited to the use of models to represent the world, it will be enough to give a counter example. Consider the work by Valentini in his (2002). Valentini showed that there are some contexts (outside of our current experimental possibilities) in which Bohm's theory and Standard Quantum Mechanics differ in their empirical predictions. To investigate how competing theories differ in their empirical predictions clearly counts as scientific work, 
and yet does not consist in using models for representation. Thus, there is more to scientific practice than the use of models for representation. ${ }^{10}$

Let us turn to the claim that theories are identified with/defined as/reduced to a class of models. At least two kinds of argument can be developed here. First, it is simply not enough to exhibit an arbitrary set of scientific models to exhibit a scientific theory. Consider for example the Aristotelian model of the cosmos, in which the Earth is at the center, the planets, including the Sun, are distributed on concentric spheres, the stars fixed on the largest sphere, with a layer of fire between us and the Moon. Put this model together with our actual model of the solar system in a set. Assuming that a minimal criterion for a theory to be scientific is that it does not generate inconsistent predictions, we can say that such a set does not constitute a theory. It follows from this that there is more to a scientific theory than being a set of models. Hence, if interpreted as including a complete account of scientific theories, then (Models) is false and the Semantic View fails. A second line of argument is the one developed by Morrison (2007, p. 201-202), which can be reconstructed as follows. According to Morrison, the Semantic View is inconsistent, because, on the one hand, it reduces theories to sets of models (thus "disposing" completely of theories), and, on the other hand, it still defines models in terms of theories. These criticisms of (Models) hold only against the strong interpretation of (Models) which takes (Models) to include a complete account of scientific theories and/or scientific practice.

One way out of these criticisms is to clearly distinguish between the various interpretations of (Models) and to explicitly commit only to the most modest one. As said earlier, the modest interpretation takes (Models) to include a partial description of the use of scientific theories in actual practice. Such a description is in turn based on factual observation. The modest interpretation of (Models) includes the claim that 1. scientific theories are typically associated with sets of models, and 2 . an important part of scientific practice consists in using these models to represent the world. The Modest Semantic View, which is the version of the Semantic View which is advocated in this paper, commits only to the modest interpretation of (Models). In doing so, the Modest Semantic View does not give an exhaustive characterization of scientific practice, even less an exhaustive account of the nature

\footnotetext{
${ }^{10}$ One could object that by studying how two theories differ in their empirical predictions, one considers models that represent the world. That need not be the case. One could study the empirical predictions of two theories, even if these theories had nothing to do with our world.
} 
of scientific theories. The Modest Semantic View commits only to a description of scientific practice which seems rather uncontroversial: an important part of the use of scientific theories in actual practice and across almost all domains of science consists in using scientific models to represent the world. The Modest Semantic View is untouched by the criticisms leveled against stronger interpretations of (Models).

\section{$3 \quad$ (Scientific $=$ Logical), the structural point of view}

The second claim of the Semantic View is the following:

$($ Scientific $=$ Logical $)-$ Scientific models can be construed as logical models.

More has to be said about the notion of a logical model. The notion of logical model appealed to here is the Tarskian notion of model (see Löwenheim (1915), Tarski (1933), see Gamut (1990) for a rigorous presentation). A logical model in this sense is a set-theoretical structure that gives an interpretation to the possible sets of sentences in which a theory is formulated so that the sentences are true on the interpretation. ${ }^{11}$ For example, consider Euclidean geometry, which can be formulated as a set of axioms. In these axioms, the terms 'point' and 'line' appear. A triangle on a plane is a structure in which what counts as a 'point' and 'line' is specified, and in which these points and lines stand in certain relationships. Within this structure, all the axioms of Euclidean geometry are true. ${ }^{12}$ Hence, the triangle on a plane is a model of Euclidean geometry. By contrast, a triangle on the surface of a sphere is a structure which gives an interpretation to the axioms of Euclidean geometry such that not all of these axioms are true within this interpretation. Hence, a triangle on the surface of a sphere is not a model of Euclidean geometry. To sum up, logical models are logical structures which are essentially characterized by their truth-making function of the corresponding theory. ${ }^{13}$

\footnotetext{
${ }^{11}$ Rigorously speaking, the interpretation is a domain along with a function assigning extensions to non-logical terms.

${ }^{12}$ It should be clear that "truth" is used here in the weak sense of "satisfaction", or "truth within an interpretation".

${ }^{13}$ Some have raised the issue of whether or not it makes any difference to characterize theories in terms of their models instead of in terms of their axioms. Given the definition
} 
With this notion of logical model in hand, one can now understand what it means for the Semantic View to take (Scientific=Logical) as one of its central claims. The Semantic View appears as a prescription to use model theory for the analysis of the scientific models. ${ }^{14}$ One important motivation is the belief that formal methods are fruitful for the systematic treatment of issues in philosophy of science and scientific methodology. It is important to emphasize that advocates of the Semantic View are committed to formal methods (see Suppes (1968, p. 656) and (2002, p. 1)).

Note that this is something that both the Semantic and the Syntactic Views share. Advocates of the Semantic View prescribe a change in tools, not in the kind of analysis that is deemed useful in philosophy of science. First order logic plus identity, advocated as the tool to use to analyze theories on the Syntactic View, is just too poor a tool to give an account of the complexities of scientific theories. The upshot is that the Syntactic View could not consider anything but oversimplified theories. Adding the axioms of (a fairly uncontroversial version of) set theory is just what is needed to be able to give an account of most important scientific results, because it includes most of mathematics (see van Fraassen (1980, chap. VII, sec. 6), Suppes $(2002$, pp. 1-2, 30, 49)). To sum up, proponents of the Semantic View advocate model theory as the correct framework for a formal analysis of scientific models. Now, let us see how such a framework can be put into use.

If the Semantic View commits to the claim that scientific models can be construed as logical models, and if logical models are essentially characterized by their truth-making function, then it must be shown that scientific models are truth-makers of a theory. Now, there are at least two ways in which the latter claim can be interpreted. One interpretation is that any scientific model, seen as a logical structure, is, by definition, true of some theory. This involves neither that we have a formulation of such a theory in hand, nor that

of logical models, it seems to make no difference. The issue was raised by Friedman (1982) and Worrall (1984). Van Fraassen (1985) gives his answer. The discussion is summarized in da Costa and French (2003, p. 30-31).

${ }^{14}$ As said before, Suppes' most general approach within the set-theoretical framework is the one discussed here, over van Fraassen's and Suppe's. Note that this implies that scientific models do not have to be mathematical structures. It suffices that scientific models be abstract structures. Any abstract structure, whether or not this structure is captured by nice mathematical equations, can be studied as such, from a model-theoretic perspective. This leaves room for an account of these sciences in which nice mathematical equations are not often possible to obtain. 
such a theory is actually used in scientific practice. Instead, the claim is only that a model-theoretic perspective can be taken on scientific models. A much stronger interpretation is to understand (Scientific=Logical) as the claim that all the models which are used to represent a given domain are truth-makers of a single and unified theory. According to this strong interpretation, the picture of how theories relate to the world would be of the following type. A high level theory is characterized by its models. Given some phenomenon in the domain of the theory, there is a scientific model which both represents the phenomenon of interest and is a logical model of the high level theory. For example, all models used in actual practice to represent the domain of Newton's theory would have to be truth-makers of Newton's laws.

It is crucial that the Semantic View explicitly rejects the strong interpretation of (Scientific=Logical) simply because the strong interpretation is easily shown to be false. This is because in most domains of scientific inquiry, the models that are used to represent the world are not truth-makers of the high-level theory. The point can be summed up in the following way. The models that are used to represent the world are typically constructed by approximating the equations of a high level theory. Some of the approximating methods used result in equations that are incompatible with the original ones. In other words, the equations of which the models are true are incompatible with the equations of the high level theory. Consequently, the models cannot be true of the high level theory. Let us make this point in a little more detail.

There are various ways in which we simplify equations in order to represent some phenomenon within scientific models. Not all of these simplifications are problematic for the strong interpretation of (Scientific=Logical). It depends on whether we consider models developed by Aristotelian abstractions, by 'Galilean idealization', or by 'mathematical impoverishment'. This has been emphasized in the literature, in particular by Redhead (1980) and Morrison (1999).

An Aristotelian abstraction ${ }^{15}$ consists in choosing the relevant variables when describing a system. Typically, the color of a sled is not considered relevant for studying its coefficient of friction, but is relevant when studying its possible success on the market. Aristotelian abstractions are not an issue for the Semantic View because the choices of the relevant variables is already made at the level of the high level theory (the color of mechanical system is

\footnotetext{
${ }^{15}$ The notion of 'Aristotelian abstraction' comes from Cartwright (1989, chap. 5).
} 
not a variable in any of Newton's equations).

A Galilean idealization ${ }^{16}$ consists in neglecting some variables or parameters that are clearly relevant to the situation studied. For example, one can decide to neglect air resistance, friction, as well as the variation in intensity of the gravitational force, when studying a sled's ability to slide down a slope. In this case, the equations of the theory are modified: what were variables in the theory become parameters set to a certain constant value (possibly zero). Consequently, the models obtained are not true of the original equations. For example, consider again Newton's laws (including the law of gravitation) as our theory of interest for studying a sled going down a slope. The model that is likely to be used to represent this phenomenon is the model of a block going down an inclined plane. Now, obviously, when going down the slope, the sled is getting closer to the center of mass of the Earth, so that, according to the law of gravitation, the intensity of the gravitational attraction that the Earth imposes on the sled increases. Typically, however, in the model of a block going down an inclined plane, the gravitational force is taken to be constant. This is incompatible with our high level theory. Consequently, the model used to represent sledding is not true of Newton's laws.

Models developed by mathematical impoverishment ${ }^{17}$ are the most problematic for the strong interpretation. Mathematical impoverishment consists in more radical modifications of the equations than Galilean idealization. Such procedures are necessary when the original equations are so complicated that it is impossible to find analytic solutions. The new equations do have analytic solutions, which we consider to be approximations of the solutions of the original equations, at least within a certain range. That said, the solutions of the approximated equations are radically different from the solutions of the original equations. One simple example of mathematical impoverishment is when one considers a polynomial development of a given function. It should be clear that the solutions of the polynomial development taken at a certain degree are far from being solutions of the original equations. The models resulting from mathematical impoverishment are radically disconnected from the high level theory.

In both cases of Galilean idealization and mathematical impoverishment, the models used to represent the domain of the high level theory are not

\footnotetext{
${ }^{16}$ The term comes from McMullin (1985).

${ }^{17}$ The term 'mathematical impoverishment' adopted here is a variation on Redhead's vocabulary in his (1980), in which he identifies the class of "impoverishment models".
} 
true of the high level theory. Hence, in general, the strong interpretation of (Scientific=Logical) is false and the Semantic View, if committed to this strong interpretation, fails. That said, the Semantic View can reject the strong interpretation of (Scientific=Logical) in favor of a more modest one, thus avoiding the difficulties above.

The modest interpretation of (Scientific=Logical) consists in the claim that the models that are used to represent some phenomenon of interest are logical models of various theories instead of a single unified one. The picture of how theories, models and the phenomena of interest relate to each other associated with the strong interpretation is rejected as too simplistic. Instead, the picture is that a whole hierarchy of models stands between the phenomena and a high level theory. In virtue of the definition of model, to the hierarchy of models corresponds a hierarchy of theories. This is not claiming that we possess or use these theories in practice. Rather, the claim is that scientific models can be considered from a model-theoretic point of view as abstract structures. Taking our previous example again, let us consider Newton's laws (including the law of gravitation) as our high level theory. To this theory correspond some models, for example, the two body gravitational system. The model of the block going down an inclined plane is not located at this level, but at a lower level. It is part of a set of models which corresponds to a theory in which the gravitational force is considered constant. Strictly speaking, this is a different theory than the theory constituted of Newton's laws along with the law of gravitation. The parallel hierarchies then continue down to the models of the experiments. ${ }^{18}$

Most advocates of the Semantic View have neither clearly drawn the distinction between the modest and the strong interpretation of (Scientific=Logical), nor explicitly rejected the later. They often seem to imply that all the models

\footnotetext{
${ }^{18}$ See also Lloyd (1994 p. 8, 13-14)[1988]. Note that this picture of a hierarchy of models meets easily Morrison's requirement in her (2007) that an adequate account of the structure of a theory be able to distinguish between the "core" of the theory and the less general models which the core theory constrains. What Morrison calls the 'theory' in her paper corresponds here to the 'high level theory'; and what she calls 'models' corresponds here to the 'lower level models'. The Modest Semantic View thus does not fall under her criticism. That said, the Modest Semantic View elaborates on this in adding that the high level theory also possesses models (in which the theory is true) and that the lower level models are true of a lower level theory. It is reasonable to believe that the "elementary way" (p. 226) in which Morrison distinguishes theories and models as being more or less "general" can be fleshed out by studying the structural relationships between high-level and low-level models in our picture.
} 
used to represent a domain are truth-makers of a single unified theory (see van Fraassen (1980, chap. 3) , Ronald Giere (1988, p. 82-83, 1999 , chap. 5, p. 92-93)). That said, it should be noted that Suppes is an important exception on this matter. He proposed the idea of parallel hierarchies of theories and models already in 1962 (1969, p. 34)[1962] :

What I have attempted to argue is that a whole hierarchy of models stands between the models of the basic theory and the complete experimental experience. Moreover, for each level of the hierarchy, there is a theory in its own right.

In agreement with the original idea of Suppes, the Modest Semantic View commits only to the modest interpretation of (Scientific=Logical), that is to say, to the claim that scientific models can be studied as abstract structures from a model-theoretic perspective. The Modest Semantic View is essentially a methodological prescription to use model theory for the formal analysis of the whole hierarchy of scientific models that are used to represent a certain domain.

\section{4 ( $\rightarrow$ Adequacy): Structure, Function, and Pragmatics}

Up to now, the Modest Semantic View appears as the methodological prescription to:

1. focus on scientific models when studying the use of scientific theories to represent the world;

2. study these models as abstract structures from a model-theoretic perspective.

What remains to be explained is what is to be accomplished with such methodological prescriptions. This is the question that ( $\rightarrow$ Adequacy) is meant to answer:

$(\rightarrow$ Adequacy $)$ - Studying scientific theories by studying their models and construing scientific models as logical models provides the means to give an adequate account of what scientists typically use to represent the world in actual practice. 
The various ways in which ( $\rightarrow$ Adequacy) can be interpreted correspond to the various levels of adequacy that one can achieve when giving an account of scientific models. The difficulty of course is to distinguish clearly between levels of adequacy and to spell out precisely what form of adequacy the Modest Semantic View can hope to achieve. ${ }^{19}$

What is needed is a characterization of what counts as 'an adequate account of scientific models', where scientific models are taken to be 'what scientists typically use to represent the world'. In order to do so, a détour from the domain of scientific theories and scientific models should prove useful. Let us try to determine first what counts as 'an adequate account of sailboats', where sailboats are taken to be 'what sailors typically use to sail'. As we shall see, at least three different kinds of account of sailboats can be distinguished. To these three kinds of account will correspond three different forms of adequacy. This analysis will finally be applied to the case of scientific models.

First, one could consider a sailboat in terms of its structure. This would consist in considering the blueprint of the sailboat, and describe what are its various parts, and how they relate to one another. On top of this, one could compare various sailboats from the point of view of their structure. Among interesting structural features of a sailboat are, for example, the ratio of length and maximal width, the rate at which the nose of the bow narrows, or the length of the keel. It should be emphasized that one can give an account of the structure of a sailboat independently of the fact that they are used to sail. The structure of sailboats can be investigated without any mention of water, currents or winds. By no means is it claimed here that such an account would be complete. However, it can be said to be adequate, even if in a narrow sense. Such an account is indeed adequate if and only if the description given of the structure of a given sailboat corresponds to the actual structure of this sailboat.

Arguably, one would require that a complete account of a sailboat contain at least, in addition to the account of the structure itself, an account of how the sailboat's structure helps serve its function. In such an account, the ratio of length and maximal width, the rate at which the nose of the bow narrows, and the length of the keel would not be studied solely as structural parts, but rather in terms of the balance between speed and stability, the capacity to break waves, and the capacity to move forward under the pressure of winds

\footnotetext{
${ }^{19}$ I wish to thank Leah McClimans for pressing me on this point.
} 
and currents. In short, one has to account for how it comes that sailboats can be used to sail: why they float, move forward with the wind, and partially resist the currents. In order to give such an account, one has to take into account these elements which are external to sailboats but are important for the use of sailboats for sailing: roughly, water, wind and currents. One has then to study the relationships between the internal structure of sailboats and these external elements such that it be explained that sailboats can be used for sailing. Such an account is adequate if and only if the relationships between the internal structure of sailboats and the external elements of the use of sailboats explain how sailboats can fit their function. Notice that one can give an adequate functional account of a sailboat without deciding on which sailboat is preferable for actual sailing, that is to say, without deciding on the pragmatics of sailboats. To answer this question one need to take into account the specific goals and means that sailors have. That said, a functional account, even if it does not account for the pragmatics of sailboats and sailing, can still be adequate, qua functional account.

Beside the structural and the functional accounts of sailboats, one can be interested in explaining the pragmatics of sailboats and sailing. To give a pragmatic account of sailboats consists in giving an account of the types of choices that sailors face and make in considering the specific goals and means they have for sailing. Goals and means vary of course, so do the choices. Typically, someone interested in racing does not make the same choices as someone interested in family vacations. According to the specific goals one has, more or less emphasis is put on speed and comfort. Notice that some considerations can also be really external to the possible performance of the boat. For example, some ecological considerations can enter into account when it comes to choose what kind of paint to use. Another obvious example of an important and highly pragmatic parameter is simply the cost of the sailboat. A pragmatic account of sailboats thus takes into account, besides the structure and the function of sailboats in general, the specific goals and means that we have when we actually choose a sailboat to sail. More precisely, a pragmatic account is expected to account for the choices made in explaining how these choices result from how the structural features of the boat, along with how these features result in specific functions, relate to the specific goals and means that sailors have. A pragmatic account hence is adequate if and only if the relationships between structure, function, goals and means are so described that the choices made are explained. 
If the analysis above is correct, then there are at least three ways in which one can give an account of sailboats - structural, functional and pragmatic, to which correspond three forms of adequacy. Let us emphasize again that the lower-level accounts can be given independently of the higher levels: structural features can be studied independently of functional or pragmatic considerations, and functional features can be studied independently of pragmatic considerations. That said, this is not the case when going down the scale of levels: functional features crucially depend on structural features, and pragmatics choices depend on both functional and structural features. To put it simply, the way sailboats are used depends on the internal make up of sailboats, and our choices over sailboats depend on both the internal make up of sailboats and how this affects their possible usage. Now, let us see how our analysis of the kinds of account and corresponding forms of adequacy one can give of sailboats applies to scientific models.

Following the analysis above, one can distinguish three ways in which to account for scientific models and three corresponding forms of adequacy. A given scientific model can be accounted for in terms of its structural features, which can further be compared with the structure of other models, independently of their function. Such a structural account is adequate if and only if the structure described is indeed the structure that the model of interest possesses. One can then study how this structure relates to typical functions of scientific models. Given that an important function of scientific models is to represent the world, an adequate functional account of scientific models will explain how the internal make up of scientific models makes it possible to use them to represent the world. A functional account hence should explain how internal features of scientific models relate to the world such that they represent the world. In short, a functional account of scientific models is adequate only if it answers the question of representation. Finally, one can study what choices scientists face and make when actually constructing and using a scientific model. Typically, scientists have to make choices concerning the relative emphasis to put on either the accuracy of the models and the tractability of the solutions. Such choices crucially depend on their specific goals and means. Cost, or epistemic accessibility (which depends on what kinds of instrument they have) are important and highly pragmatic considerations. A pragmatic account of scientific models is then adequate if and only if the structure of the models along with how this structure makes the model serve specific functions are related to the goals and means of scientists such that typical choices that these scientists make are explained. 
The three ways in which ( $\rightarrow$ Adequacy) can be interpreted should now be clear. The Modest Semantic View commits only to the claim that studying scientific theories from the point of view of scientific models, and studying scientific models from a model-theoretic point of view, is sufficient to give an adequate account of scientific models in the structural sense. Stronger interpretations of ( $\rightarrow$ Adequacy) consists in the claims that studying scientific theories from the point of view of scientific models, and studying scientific models from a model-theoretic point of view, is sufficient to give an adequate account of the function or of the pragmatics of scientific models (or both). It should be recognized that the focus on structure has been consistent in the Semantic View literature since its inception. That said, it is not clear that advocates of the Semantic View have ever made the above tripartite distinction explicit. They often seem quite ambitious as to what the Semantic View can help us accomplish. The early van Fraassen (1980) hopes to formally characterize empirical adequacy, a theory-world relation, in terms of isomorphism..$^{20}$ In his (1989), he hopes that the Semantic View could allow us to give answers to the problems of scientific explanation, confirmation and modality.

In the case of ( $\rightarrow$ Adequacy) as in the case of (Models) and of (Scientific $=$ Logical), it is crucial to make the distinctions between the modest and stronger interpretations clear, to explicitly reject the stronger ones, and to commit only to the modest one. This is because, again, the stronger ones are easily shown to be false, so that if the Semantic View commits to any of these stronger interpretations, then it simply fails. As it is explained in the following, to study scientific models as abstract structures does not allow one to give an adequate account either of the function or of the pragmatics of these models.

It has been repeatedly pointed out in the literature that the Semantic View cannot give an adequate functional account of scientific models. ${ }^{21}$ As

\footnotetext{
${ }^{20}$ Later on, van Fraassen became well aware of the problem of structuralism as van Fraassen (2006) shows. That said, he does not explicitly conclude from this that the scope of the Semantic View should be restricted.

${ }^{21}$ Even if Cartwright $(1983,1989)$ has been developing similar views over the past twenty years, Morrisson (1999) is often cited for posing the criticism of the Semantic View along these lines. Thomson-Jones (2006) gives an analysis of the relation between logical models and scientific models, the former being characterized by their truth making function, and the latter by their representational function. Brading and Landry (2006) and Frigg (2006) gives also a systematic analysis of the problem. It should be noted that van Fraassen (2006) presents the same problem in referring to Reichenbach (1965). All point out that the formal
} 
noted earlier, it should be rather uncontroversial that an essential function of scientific models is to represent the world. Consequently, a functional account of scientific models cannot be adequate unless it at least addresses the issue of how these models represent the world. Now, what does the representational function of scientific models consist in? A minimal characterization of representation is the following: it is a relation between a representans and a representandum. In our case, the representans is a scientific model, while the representandum is a phenomenon in the world. Now, we have seen that, within the Semantic View, one construes scientific models as abstract formal structures and uses model theory to study their structural properties as well as their structural relationships with other formal structures. The tools that the Semantic View offers, that is, logical models and model theory, are adequate tools only to study formal relationships between formal structures. The problem is of course that the world is not a formal structure. Consequently, the issue of how scientific models represent the world, that is, the question of how abstract formal structures relate to non-abstract, non-formal things such that 'representation' occurs simply cannot be addressed with the tools proposed. So, the Semantic View does not give the means for an adequate account of the representational function of scientific models. The upshot is: if $(\rightarrow$ Adequacy) is taken as the claim that the Semantic View does give the means for such an account, then the Semantic View fails.

It has been pointed out in the literature that taking the Semantic View on scientific models does not give the means to give an adequate pragmatic account of scientific models either. ${ }^{22}$ Recall that, in order to give an adequate pragmatic account of scientific models, one has to explain the choices that scientists make over scientific models in relation to their specific goals and means. In other words, one has to understand how certain types of scientific models (characterized by certain structural features and related functions) are chosen depending on certain goals and means. Now, most of model construction consists in deciding over which approximations are appropriate. The problem is that there is no formal justification for these approximations. Even less is there any formal justification for which approximation is preferable in regards to the scientists' means and goals. Of course, knowledge of the formal structure of the various possible models that could be adopted

features of logical models are not sufficient to account for the essential representational function of scientific models.

${ }^{22}$ Redhead makes the point in his (1980). Here again, Morrison (1999, p. 39) develops this point extensively. 
is an important part of the decision process. Back to sailboats, the choice over which kind of boat is the most appropriate for someone depends on the various possible structures that sailboats can have. Similarly, the choice over which kind of model is the most appropriate for someone depends on the possible internal structure that models can have. That said, a structural account is not sufficient. Within an adequate pragmatic account, the various possible structures have to be understood in so far as how they relate to specific pragmatic considerations. Just as in the case of the question of representation, the Semantic View fails to give the appropriate tools to account for such relationships simply because such these relationships are not formal relationships between formal structures. Consequently, if ( $\rightarrow$ Adequacy) is taken as the claim that the Semantic View does give such means, then the view fails.

To make the argument in favor of the Modest Semantic View, it remains to show that the tools proposed by the Modest Semantic View are appropriate to give an adequate structural account of scientific models. No one has said it better than Suppes (Suppes 1960, p. 17):

[... the notion of model in the sense of logicians provides the appropriate intellectual tool for making the analysis both precise and clear.

In particular, it makes the notion of structure and of structural relationships between models precise. Such a precise and well-defined notion is useful in addressing both issues of internal structure of theories and interrelations between theories. For example, van Fraassen (1991) uses the method presented in his (1989), to examine the controversies that have plagued the philosophy of quantum mechanics since the inception of the theory. He offers a formal presentation of the issue of whether or not quantum phenomena can be described by deterministic causal models. Lloyd has shown how the semantic conception is an interesting framework in which to study population genetics. Within the framework of the Semantic View, Lloyd offers a categorization of the various mathematical models in population genetics, leads a discussion of units of selection based on this analysis, and offers a systematic analysis of types of confirmation based on the investigation of the relationships between data models and theoretical models. Focussing on the structures of these models, she is, for example, able to distinguish between various kinds 
of group selection, and to argue against genic selectionism. ${ }^{23}$ Finally, in his (2002), Suppes shows how proving representation theorems with model theory is useful to address the question of reductionism. To prove a representation theorem is to show that there is a specific class $S$ of models such that every model of a theory $T$ is isomorphic to a member of $S$. In other word, all the models of the theory are "represented" by a model in $S$. This is arguably an interesting way to formalize the possible reduction of a theory $T$ to the theory $T^{\prime}$ associated with the restricted class $S$ of models. ${ }^{24}$ In general then, model theory is an appropriate formal tool for the systematic investigation of the structure of theories and the interrelations between theories, and thus for giving an adequate structural account of scientific models.

To sum up; three interpretations of ( $\rightarrow$ Adequacy) have been distinguished. The modest interpretation of ( $\rightarrow$ Adequacy) consists in the claim that studying scientific practice and scientific theories from the point of view of scientific models, and studying scientific models from a model-theoretic point of view, allows one to give an adequate structural account of what scientists typically use to represent the world. Stronger interpretations include the claims that the method proposed allows one to give an adequate functional account, or an adequate pragmatic account of scientific models, or both. The objections that have been leveled against the Semantic View in the recent literature have been rehearsed, and have been shown to hold only under the strong interpretations of ( $\rightarrow$ Adequacy). That said, it has been shown that there is an interpretation of ( $\rightarrow$ Adequacy) under which $(\rightarrow$ Adequacy) escapes these objections. The Modest Semantic View only commits to the claim that studying scientific models as formal, abstract structures gives the means to give an adequate account of the structure of what scientists typically use to represent the world.

\footnotetext{
${ }^{23}$ It should be noted that Lloyd's analysis does not include the assumption that population genetics is all there is to evolutionary theory. Rather, she aims at giving an account of the structure of evolutionary theory in a broader sense, including issues about species formation and extinction, group selection, and the tempo and mode of selection (1994, chap. 1 note 4 . and chap. 3)[1988].

${ }^{24}$ One final note: an important advantage of the Modest Semantic View is that it does not entail any position in the realist/antirealist debate. This follows from our argument that the Semantic View does not provide the appropriate tools for giving an account of the representational function of scientific models. It should also be clear from the consideration that there are advocates of the Semantic View on both sides of the debate: van Fraassen on the empiricist side, Suppe, Giere and da Costa and French on the realist side.
} 


\section{Conclusion: The Modest Semantic View}

The Modest Semantic View (MSV) consists in the following claims:

- (Models) - Scientific theories and scientific practice can be partially characterized by the scientific models that scientists typically use to represent the world;

- $($ Scientific $=$ Logical $)$ - Scientific models can be studied as abstract structures from a model-theoretic point of view;

- $(\rightarrow$ Adequacy $)$ - Studying scientific theories by studying their models and construing scientific models as logical models provides the means to give an adequate structural account of what scientists typically use to represent the world in actual practice. .

The first two claims taken together amount to a methodological prescription: it indicates what tools are proposed within MSV. Holding the first claim implies holding a partial descriptive claim about scientific practice: scientists typically use scientific models to represent the world. Such a claim does not amount to a complete account of either scientific representation, scientific practice, or scientific theories. The second claim is essentially the methodological prescription to use model theory for the systematic investigation of scientific models construed as abstract structures. The claim is not that all scientific models used to represent a given domain are truth-makers of a single unified theory of the domain. The naive picture of how theories, models and phenomena relate to each other is replaced by the more realistic picture that a whole hierarchy of sets of models and corresponding theories stand in between high level theories and the data models. The last claim is a claim of adequacy: it is stated that the method proposed allows one to give an adequate structural account of what scientists typically use to represent the world. The form of adequacy that MSV can achieve is rather narrow: MSV does not provide the appropriate tools to account either for the function or for the pragmatics of scientific models. Nor is it supposed to.

There is no question that scientific models have a representational function. Nor is there any doubt that the pragmatics of modeling is an important domain of investigation for philosophy of science. If the Semantic View is supposed to be a comprehensive view of science, then it had better say something about the functional features and the pragmatics of scientific models. 
MSV does not pretend to be a complete account of scientific theories and scientific practice. This is consistent at least with the thought of one of the founding fathers of the Semantic View (Suppes (1994, p. 214)):

It is a myth engendered by philosophers - even in the past to some extent by myself - that the deductive organization of physics in nice set theoretical form is an achievable goal. A look at the chaos in the current literature in any part of physics is enough to quickly dispel that illusion, this does not mean that set theoretical work cannot be done, it is just that its severe limitations must be recognized.

According to MSV, the severe limitations that have to be recognized are just that the Semantic View cannot give either a functional or a pragmatic account of scientific models. That said, just as Suppes remarks: this does not mean that no set theoretical work can be done, or that it is not useful. Given that any functional or pragmatic account of scientific models depend on the structural features of these models, the structural account that MSV gives is a crucial component of any full account of scientific theories and scientific practice.

\section{References}

[1] Balzer Wolfgang, C. Ulises Moulines and Joseph D. Sneed (1987). An Architectonic for Science: The Structuralist Program, Dordrecht: Reidel.

[2] Beatty, John (1979). Traditional and Semantic Accounts of Evolutionary Theory, Ph.D. Dissertation, Indiana University.

[3] Beatty, John (1981). "What's Wrong with the Received View of Evolutionary Theory?", in P. Asquith and Ronald Giere (eds.), PSA 1980, 2: 397-426, East Lansing, MI: Philosophy of Science Association.

[4] Beth, Evert W. (1948a). "Analyse Semantique des Theories Physiques", Synthese, 7: 206-207.

[5] Beth, Evert W. (1948b). Natuurphilosophie, Gorinchem: Noorduyn. 
[6] Beth, Evert W. (1949). "Towards an Up-to-date Philosophy of the Natural Sciences," Methodos, 1: 178-185.

[7] Beth, Evert W. (1960). "Semantics of Physical Theories", Synthese, 12 : 172-175.

[8] Brading, Katherine and Elaine Landry (2006). "Scientific Structuralism: Presentation and Representation", Philosophy of Science, 73: 571-581.

[9] Carnap, Rudolph (1966). Philosophical Foundations of Physics, New York: Macmillan.

[10] Cartwright, Nancy D. (1983). How the Laws of Physics Lie, Oxford: Oxford University Press.

[11] Cartwright, Nancy D. (1989). Nature's Capacities and their Measurement, Oxford: Oxford University Press.

[12] Cartwright, Nancy D. (1999). "Models and the Limits of Theory: Quantum Hamiltonians and the BCS Model of Superconductivity", in Morgan and Morrison (1999): 241-281.

[13] Churchland, Paul M. and Clifford A. Hooker (1985) (ed.). Images of Science: Essays on Realism and Empiricism, with a Reply from Bas C. van Fraassen, Chicago: University of Chicago Press.

[14] Colodny, Robert G. (ed.) (1972). Paradigms and Paradoxes: The Philosophical Challenge of the Quantum Domain, Pittsburgh: Pittsburgh University Press.

[15] Corcoran, John (1983) (ed.). Tarski: Logic, Semantics, Metamathematics, papers from 1923 to 1938. Indianapolis: Hackett Publishing Company.

[16] da Costa, Newton C.A. and Steven French (2003). Science and Partial Truth: A Unitary Approach to Models and Scientific Reasoning, Oxford: Oxford University Press.

[17] Ereshefsky, Marc (1991). "The Semantic Approach to Evolutionary Theory", Biology and Philosophy, 5: 7-28. 
[18] Friedman, Michael (1982). "Review of the scientific image", Journal of Philosophy 79(5): 274-283.

[19] Friedman, Michael (1999). Reconsidering Logical Positivism, Cambridge: Cambridge University Press.

[20] Frigg, Roman (2006). "Scientific Representation and the Semantic View of Theories", Theoria 55: 49-65.

[21] Frigg, Roman and Stephan Hartman (2006). "Models in Science", Stanford Encyclopedia of Philosophy, in Edward N. Zalta (ed.), http://plato.stanford.edu/archives/spr2006/entries/ models-science/.

[22] Gamut, L.T.F. (1990). Logic, Language and Meaning: Introduction to Logic, Chicago: University of Chicago Press.

[23] Giere, Ronald (1979). Understanding Scientific Reasoning, New York: Holt, Rinehart and Winston.

[24] Giere, Ronald (1988). Explaining Science, Chicago: University of Chicago Press.

[25] Giere, Ronald (1999). Science without laws, Chicago: University of Chicago Press.

[26] Hempel, Carl (1963). "Implications of Carnap's work for the philosophy of science" in Paul Schilpp (1963): 685-709.

[27] Hughes, R.I.G. (1989). The Structure and Interpretation of Quantum Mechanics, Cambridge: Harvard University Press.

[28] Humphreys, Paul (1994) (ed.). Patrick Suppes : Scientific Philosopher, II, Theory, Structure and Measurement Theory, Dordrecht: Kluwer Academic.

[29] Krause, Décio and Bueno, Otávio (2008) "Scientific Theories, Models, and the Semantic Approach", preprint http://philsci-archive. pitt.edu/archive/00003958/, forthcoming in Principia, 2008. 
[30] Löwenheim, Leopold (1915). "Über Möglichkeiten im Relativkalkül", Mathematische Annalen, 76: 447-470, eng. transl. "On Possibilities in the Calculus of Relatives" in van Heijenoort(1967): 228-251.

[31] Lloyd, Elisabeth A. (1994)[1988]. The Structure and Confirmation of Evolutionary Theory, Princeton: Princeton University Press.

[32] Morgan, Mary S. and Margaret Morrison (1999). Models as Mediators, Cambridge: Cambridge University Press.

[33] Morgenbesser Sidney (ed.) (1967). Philosophy of Science Today, New York: Basic Books.

[34] Morrison, Margaret (1998).

[35] Morrison, Margaret (1999). "Models as Autonomous Agents" in Morgan and Morrison (1999): 38-65.

[36] Morrison, Margaret (2007). "Where have all the theories gone?", Philosophy of Science, 74: 195-228.

[37] Moulines, C. Ulises (1975). "A Logical Reconstruction of Simple Equilibrium Thermodynamics", Erkenntnis, 9: 101-130.

[38] Nagel, Ernest, Patrick Suppes, and Alfred Tarski (1962) (ed.). Logic, Methodology and Philosophy of Science, Proceedings of the 1960 International Congress, Stanford: Stanford University Press

[39] Niiniluoto, Ilkka and Raimo Tuomela (eds.) (1979). The Logic and Epistemology of Scientific Change, Amsterdam: North Holland.

[40] Parrini, Paolo, Merrilee Salmon et Wesley C. Salmon (ed.) (2003). Logical Empiricism: Historical and Contemporary Perspectives, Pittsburgh: University of Pittsburgh Press.

[41] Putnam, Hilary (1962)[1979] "What Theories Are Not," in Nagel et al. (1962). Reprinted in H. Putnam, Mathematics, Matter and Method: Philosophical Papers, vol. 1. Cambridge: Cambridge University Press, 1979: 215-227.

[42] Redhead, Michael (1980). "Models in Physics," British Journal for Philosophy of Science 31: 145-163. 
[43] Reichenbach, Hans (1965)[1920]. The Theory of Relativity and A Priori Knowledge, Trad. Maria Reichenbach, Berkeley: University of California Press.

[44] Schilpp, Paul (ed.) (1963). The Philosophy of Rudolph Carnap, La Salle (III): Open Court Publishing.

[45] Sneed, Joseph D. (1971). The Logical Structure of Mathematical Physics, Dordrecht: Reidel.

[46] Stegmüller, Wolfgang (1976). The Structure and Dynamics of Theories, New York: Springer Verlag.

[47] Stegmüller, Wolfgang (1979). "The Structuralist View: Survey, Recent Developments and Answers to Some Criticisms", in Niiniluoto and Tuomela (1979).

[48] Suárez, Mauricio (2003). "Scientific Representation: Against Similarity and Isomorphism." International Studies in Philosophy of Science, 17: 225-244.

[49] Suppe, Frederick (1974). "Some Philosophical Problems in Biological Speciation and Taxonomy", in Wojcieckowski (1974): 190-243.

[50] Suppe, Frederick (1977a). "The Search for Philosophical Understanding of Scientific Theories", in Suppe (1974b): 3-232.

[51] Suppe, Frederick (1977b) (ed.). The Structure of Scientific Theories, Champain (Ill.): University of Illinois Press.

[52] Suppe, Frederick (1979). "Theory Structure", in Current Research in Philosophy of Science, East Leansing, MI: Philosophy of Science Association: $317-338$.

[53] Suppe, Frederick (1989). The Semantic Conception of Theories and Scientific Realism, Urbana: University of Illinois Press.

[54] Suppes, Patrick (1960). "A Comparison of the Meaning and Uses of Models in Mathematics and the Empirical Sciences", Synthese 12: 287301, reprinted in Suppes (1969): 10-23. 
[55] Suppes, Patrick (1962). "Models of data", in Nagel et al. (1962), reprinted in Suppes (1969): 24-35.

[56] Suppes, Patrick (1967). "What is a Scientific Theory?" in S. Morgenbesser (1967): 55-67.

[57] Suppes, Patrick (1968). "The Desirability of formalization in science", Journal of Philosophy, 65:651-664.

[58] Suppes, Patrick (1969), Studies in the Methodology and Foundations of Science: Selected Papers from 1951 to 1969, Dordrecht: Reidel.

[59] Suppes, Patrick (1994) answer to Wójcicki in Humphreys (1994): 146149.

[60] Suppes, Patrick (2002), Representation and Invariance of Scientific Structures, CSLI Lecture Notes, Stanford : Center for the Study of Language and Information.

[61] Tarski, Alfred (1933). "The Concept of Truth in the Languages of the Deductive Sciences" expanded English translation in Corcoran (1983): 152-278.

[62] Thompson, Paul (1989). The Structure of Biological Theories. Albany: SUNY Press.

[63] Thomson-Jones, Martin (2006). "Models and the Semantic View", Philosophy of Science, 73: 524-535.

[64] Valentini, Anthony (2002). "Signal-Locality in Hidden-Variables Theories", Physics Letters A, 297: 273-278.

[65] van Fraassen Bas C. (1972). "A Formal Approach to the Philosophy of Science", in Colodny (ed.) (1972).

[66] van Fraassen Bas C. (1980). Scientific Image, Oxford: Clarendon Press.

[67] van Fraassen, Bas. C. (1985). "Empiricism in the philosophy of science", in Churchland and Hooker (1985): 245-308.

[68] van Fraassen, Bas C. (1989). Laws and Symmetry, Oxford: Clarendon Press. 
[69] van Fraassen, Bas C. (1991). Quantum Mechanics, An Empiricist View, Oxford: Oxford University Press.

[70] van Fraassen Bas. C. (2006). "Representation: the Problem for Structuralism", Philosophy of Science, 73: 536-547.

[71] van Heijenoort, Jean (1967) (ed.). From Frege to Gödel: A Source Book in Mathematical Logic, 1879-1931, Cambridge, MA : Harvard University Press.

[72] Wojcieckowski J.A. (ed.) (1974). Conceptual Basis of the Classification of Knowledge, Munich: Verlag Dokumentation.

[73] Worrall, John (1984). "An unreal image", The British Journal of Philosophy of Science, 35(1): 65-80. 